\title{
Globe
}

Revue internationale d'études québécoises

\section{Karim Larose : La langue de papier. Spéculations linguistiques \\ au Québec (1957-1977). Montréal, Presses de l'Université de Montréal, 2004}

\section{François Paré}

Volume 8, numéro 2, 2005

URI : https://id.erudit.org/iderudit/1000921ar

DOI : https://doi.org/10.7202/1000921ar

Aller au sommaire du numéro

Éditeur(s)

Globe, Revue internationale d'études québécoises

ISSN

1481-5869 (imprimé)

1923-8231 (numérique)

Découvrir la revue

Citer ce compte rendu

Paré, F. (2005). Compte rendu de [Karim Larose : La langue de papier. Spéculations linguistiques au Québec (1957-1977). Montréal, Presses de l’Université de Montréal, 2004]. Globe, 8(2), 307-310.

https://doi.org/10.7202/1000921ar d'utilisation que vous pouvez consulter en ligne.

https://apropos.erudit.org/fr/usagers/politique-dutilisation/ 
RECENSIONS

\section{Karim Larose}

La langue de papier. Spéculations linguistiques

au Québec (1957-1977).

Montréal, Presses de l'Université de Montréal, 2004.

C'est sur la langue française, son statut politique et son avenir en Amérique qu'ont porté certains des plus grands essais de la seconde moitié du $x^{e}$ siècle au Québec. Des tribuns et des auteurs importants lui ont consacré l'essentiel de leurs interventions publiques et de leurs écrits. La langue, synecdoque par excellence de la nation, constitue à la fois l'horizon dramatique et le lieu heuristique où ont circulé et se sont entrecroisés les discours de l'inquiétude à la confluence même du littéraire et du politique. C'est pourquoi il fallait une bonne part de témérité pour tenter de saisir, comme le fait magistralement ici Karim Larose, la substance de cette agitation qui sert de toile de fond à toute une époque de profondes et intenses transformations. Larose nous offre une galerie de portraits d'hommes et de femmes (d'hommes surtout!) qui, durant les années 1960, ont fait du débat sur la langue le cœur de leur réflexion politique et de leur écriture.

De Raymond Barbeau et Jacques Ferron à Jean-Marie Laurence et Léandre Bergeron, Larose ne cherche pas à recenser les confortables évidences qui meublent la culture québécoise contemporaine et son interprétation conventionnelle de la Révolution tranquille. Voulant dépasser les discours d'apparat, l'auteur nous invite, à l'instar d'André Belleau, à "surprendre les voix" en départageant "ce qui, dans la réflexion sur la langue, définit le bruit et la parole, ce qui peut être entendu et ce qui ne l'est pas encore, ce qui, du mutisme, peut être porté au témoignage. (p. 25). La lecture méticuleuse qui ressort de cette approche de l'u archive " et du " déchet " force un retour passionnant aux portes de la Révolution tranquille. À ceux ou celles qui douteraient aujourd'hui de l'existence même de cette période de vives mutations, le travail de Larose présente le tableau saisissant d'un bouillonnement intellectuel dans lequel se sont engagées, jusqu'à s'y perdre, certaines des voix les plus lucides et les plus complexes de la fin du siècle dernier au Québec.

Larose retrace la mise en place, au sortir des années 1950, d'une conception "expressiviste " de la langue qui structurera l'œuvre de nombreux écrivains et animateurs politiques. S'opposant à une vision 
instrumentaliste du français, souvent associée à l'américanisation du continent et à l'anglicisation, l'expressivisme linguistique s'appuiera sur les liens congénitaux entre le sujet psychologique et sa langue "natale". C'est pourquoi, envisagée sur le plan de la décolonisation, la langue pourra d'autant moins s'accommoder du bilinguisme proposé par les autorités fédérales que cette conception double de l'appartenance linguistique apparaîtra à plusieurs - dont le plus célèbre est sans doute Gaston Miron - comme une déchirure dans le tissu existentiel de la culture d'origine. Avant Miron, André d'Allemagne, traducteur de formation, avait dénoncé le bilinguisme fédéral comme une forme déguisée de l'unilinguisme affiché par la société anglo-canadienne dominante.

La figure de Jacques Ferron domine entièrement le travail de Larose. Si certains porte-parole ont pu annoncer les débats à venir, c'est chez l'auteur de La nuit que se résument, dès 1958, les premiers fantasmes d'un Québec conçu comme un "État français unilingue *. Ce programme fort, qui deviendra en une dizaine d'années une véritable orthodoxie politique, s'appuie sur les premiers écrits de Ferron, repris par ses proches, tels Raoul Roy à la Rerue socialiste et, plus tard, Raymond Barbeau et André d'Allemagne à l'Alliance Laurentienne. Larose fait voir avec précision l'enchevêtrement des domaines littéraire et politique au moment où se publient une myriade d'éphémères petits journaux socialistes et indépendantistes. À l'indépendance politique du Québec se substituera donc la souveraineté absolue de la langue française, considérée comme seule capable de rendre compte de l'histoire des Québécois et des processus d'affranchissement qu'il convient de mettre en œuvre.

L'ouvrage de Larose ne s'en prend jamais à la validité du concept d'un Québec unilingue français, mais il en dessine l'histoire et en montre les charnières idéologiques. C'est dans ce contexte qu'il analyse la parution du numéro de Liberté sur la langue en 1964, les textes cruciaux de Pierre Elliott Trudeau et d'Hubert Aquin sur le Canada français, l'action de Gérald Godin au sein de Parti pris, le conflit larvé entre André Major et Claude Jasmin sur l'émergence du joual comme langue littéraire. Dans l'ensemble de ces débats, Larose retrouve la proposition ferronienne de la complétude linguistique : " une langue complète n'est telle que parce qu'elle renferme en son sein la totalité du savoir humain possible, parce qu'elle donne à son locuteur un accès à l'éventail "complet" des moyens d'expression. (p. 158). La souveraineté des individus et des nations passe ici, on le voit bien, par la mise en place 
de dispositifs linguistiques qui assurent au français l'accès à l'ensemble des champs d'expression dans l'espace public.

Dans un dernier chapitre d'une grande importance, Larose suit à la trace les idées maîtresses de "langue natale * et de " parole * chez deux essayistes et poètes qui ont consacré de nombreux textes à la question du français au Québec. Chez Gaston Miron, d'abord, Larose soulève l'arrimage des propos sur la langue à une vision déficitaire de l'histoire québécoise. C'est chez Miron que l'expressivisme linguistique trouve ses échos les plus puissants : “ Dès 1957, tout se passe dans la réflexion de Miron comme si le défaut de parole ne pouvait que diriger l'homme, perdu au milieu de signes inatteignables, vers un expressivisme où il ne peut que se considérer en position d'humilité devant l'immensité de la langue " (p. 318). Cette faille identitaire, qui mènera à une condamnation sans équivoque du bilinguisme, explique la recherche chez Miron d'une société capable d'affirmer l'homogénéité de sa culture et de sa langue. Larose indique que cette vision réductrice est aujourd'hui intenable ; il n'en reste pas moins qu'elle est toujours perceptible dans une large part du discours intellectuel au Québec. Comparant Miron à Hölderlin, Larose livre ses pages les plus inspirantes, notamment sur le nativisme des positions linguistiques de Miron : "Le natal serait ainsi l'espace de langage où, déployée, la langue s'étend sans limites assignées, où l'on a du jeu pour parler, où par conséquent la prise de distance devient possible. (p. 335). À son tour, l'essayiste qu'est Larose s'inscrit dans la lignée de ses prédécesseurs, s'appuyant sur la langue elle-même afin de créer des possibilités de distance et d'ouverture.

Enfin, c'est chez Jacques Brault que Larose trouve vraisemblablement les textes les plus fraternels. En effet, si, dès les années 1950, Brault se fait l'apologiste de Miron, il s'en détache de façon subtile dans ses essais subséquents. Aussi fait-il de la traduction un processus dynamique qui permet de résoudre les tensions aliénantes. Loin du " traduitdu * paralysant dénoncé dans les textes didactiques de L'bomme rapaillé, Brault relève les "espèces de postulats " qui empêchent d'être à l'écoute des "voix lointaines toutes proches " au sein du discours. Larose tire de ce dialogue entre Miron et Brault la leçon d'une époque d'extraordinaires spéculations sur la langue, ouvrant enfin sur l'errance et la différence.

Par l'ampleur décisive de sa perspective critique et par l'éclectisme de son érudition, le livre de Karim Larose me semble une lecture 
essentielle. Toute considération sur la langue doit donner lieu à de " délicats équilibres " et à de " discrètes épiphanies ", écrit l'auteur dans les dernières lignes de l'ouvrage. Cette contingence lumineuse, éclairant toute une époque, lui aura permis de comprendre le sujet de la langue au Québec en préservant son essentielle dignité et sa modernité.

François Paré University of Waterloo

\section{André Lamontagne}

Le roman québécois contemporain.

Les voix sous les mots.

Montréal, Fides, coll. "Nouvelles études québécoises ", 2004.

Où en est le roman québécois? Quelle est sa situation, sa valeur? Qu'est-ce qui l'habite, l'occupe, le secoue, le hante ? En quoi se distinguet-il ? Bref, comment se porte la littérature québécoise contemporaine ? La question est 'certes d'actualité, mais le manque de recul exige une certaine prudence à l'égard des jugements prononcés. Peut-être est-ce d'ailleurs la raison pour laquelle, depuis 1980 , la critique se contente généralement de souligner la diversité de la production romanesque : s'il existe un quelconque dénominateur commun, c'est celui de l'hétérogénéité. Autrement, le roman québécois résiste à toute catégorisation. Pourtant, aussi kaléidoscopique qu'apparaisse le panorama littéraire québécois, il semble que ce soit là éviter de plonger trop en avant dans le débat. Qu'il y règne la disparité, certes - c'est le lot de toute littérature contemporaine dont on comprend encore difficilement les enjeux-, mais qu'il ne s'y exprime aucune préoccupation ou esthétique communes, la chose parait peu probable.

Aussi le vent tend-il à changer. Avec le $\mathrm{xxI}^{\mathrm{e}}$ siècle vient le temps des bilans - et des prédictions. Des critiques littéraires osent régulièrement quelque tentative de classification. Des colloques témoignent de l'intérêt de et à l'égard de la production récente. Des ouvrages d'envergure sont publiés, chacun saisissant la littérature contemporaine par un trait récurrent et définitoire - Roseline Tremblay poursuit le travail d'Ảndré Belleau 